\title{
Targeted rehabilitation reduces visual dependency and improves balance in severe traumatic brain injury: a case study
}

\section{Diego Kaski, Joseph Buttell \& Richard Greenwood}

To cite this article: Diego Kaski, Joseph Buttell \& Richard Greenwood (2017): Targeted rehabilitation reduces visual dependency and improves balance in severe traumatic brain injury: a case study, Disability and Rehabilitation, DOI: 10.1080/09638288.2016.1276976

To link to this article: http://dx.doi.org/10.1080/09638288.2016.1276976

Published online: 23 Jan 2017.

Submit your article to this journal $[\pi$

a)

View related articles ¿

View Crossmark data $\nearrow$ 


\title{
Targeted rehabilitation reduces visual dependency and improves balance in severe traumatic brain injury: a case study
}

\author{
Diego Kaski ${ }^{a}$, Joseph Buttell ${ }^{b}$ and Richard Greenwood ${ }^{a, b}$ \\ ${ }^{\mathrm{a}}$ Institute of Neurology, University College London, London, UK; ${ }^{\mathrm{b}}$ Regional Neurological Rehabilitation Unit, Homerton University Hospital, \\ London, UK
}

\begin{abstract}
Purpose: To further understand the mechanisms underlying gait impairment following traumatic brain injury.

Case report: A 58-year-old man presented with marked unsteadiness and motion sensitivity following a severe traumatic brain injury. He underwent a 6-week inpatient rehabilitation program focused on reweighting and subsequently re-integrating ascending interoceptive information, by gradual reduction of maladaptive visual fixation techniques. We report clinical neurological outcomes and measures of functional outcome, as well as an objective assessment of visual dependency (the rod and disk test) at baseline and after the rehabilitation.

Results: Clinically, the patient had gait unsteadiness exacerbated by visual motion. A significant reduction in visual dependency occurred with tailored multi-disciplinary rehabilitation via gradual removal of visual fixation strategies that the patient had developed to maintain balance ( $t$-test; $p<0.01$ ), as well as clinical improvements in gait, balance, and functional outcome.

Conclusions: We highlight the importance of visual dependency in the generation of maladaptive gait strategies following brain injury. Our data suggest assessing and treating visual dependency to be an important component of gait rehabilitation after traumatic brain injury.

- IMPLICATIONS FOR REHABILITATION

- Whilst gait disturbance in TBI is multifactorial, abnormal visual dependency may be important but under-recognised component of the disorder.

- Visual dependency can be easily and objectively assessed by the bedside in patients using a dynamic rod and disc test.

- Tailored rehabilitation with gradual reduction of maladaptive visual fixation can reduce visual dependency and contribute to improved gait and balance following TBI.
\end{abstract}

\section{Introduction}

Balance and gait impairments are common physical symptoms following brain trauma of all severities [1-4], but an understanding of the underlying neural mechanisms remains incomplete. Gait impairment after TBI may result from impairment in a number of sensory modalities, and musculoskeletal damage related to the injury. In accordance with the CAse REports checklist, we present a patient who sustained a severe traumatic brain injury and developed severe unsteadiness. We discuss the mechanisms underlying his postural imbalance, with a focus on abnormal visual dependency, which improved following targeted physical therapy.

\section{Case report}

A 58-year-old man was admitted to a neurosurgical unit following a severe head injury sustained as a result of a nine foot fall, after which he was found unconscious by his wife. His admission Glasgow Coma Score was 8/15. Acute imaging revealed a left subdural parietal hematoma, left hemispheric subarachnoid blood, and a left ulnar fracture. There was no skull fracture, and no microhaemorrhages on MRI. He was managed conservatively in a high-dependency setting. He regained full consciousness within $24 \mathrm{~h}$, but had a post-traumatic amnesia of approximately two weeks. One week into his admission he described "dizziness" manifest as rotational vertigo and oscillopsia on head movement. Neuro-otological assessment revealed multiple canal BPPV, which was treated successfully with serial repositioning maneuvers for the affected semicircular canals. He received in-patient general physiotherapy whilst awaiting transfer to a specialist rehabilitation unit but his balance remained severely impaired.

Neurological examination on admission to the rehabilitation unit 8 weeks post-injury was normal apart from an apraxic gait, with large amplitude, multi-directional, sudden and jerky postural adjustments, particularly in response to people walking towards him and following active head movements, suggestive of abnormal visual dependency. His stance was more impaired with eyes open - versus eyes closed - when sudden large antero-posterior trunk movements, and violent arm movements would occur. His gait was alarming to observers with an apparent high risk of falling, reminiscent of functional neurological gait disorders [5]. He fiercely contested any impairment in balance, he had no neglect, 


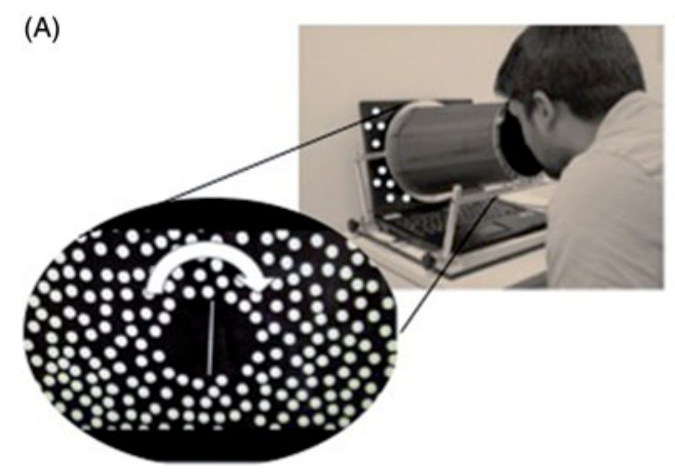

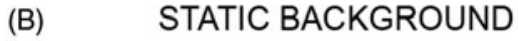

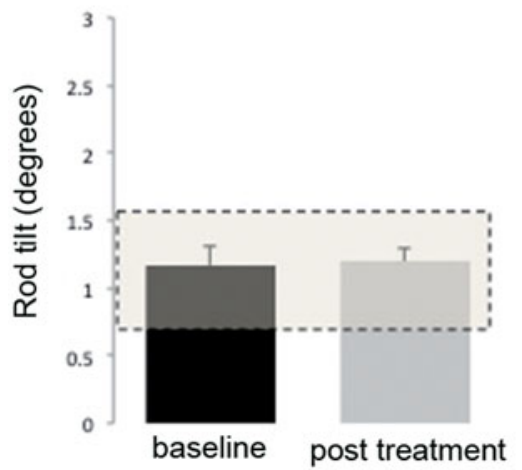

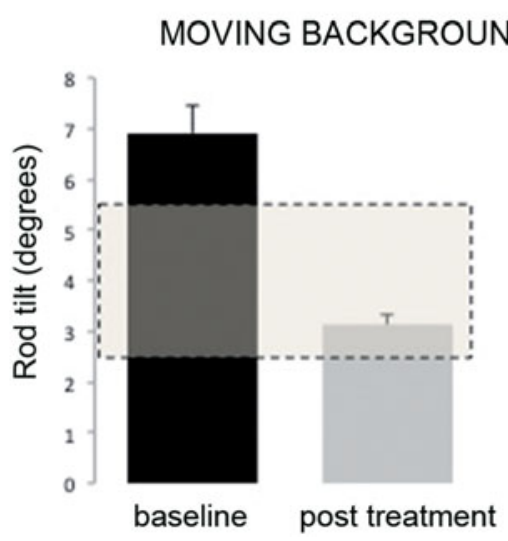

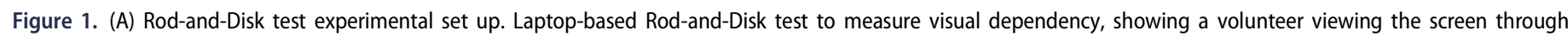

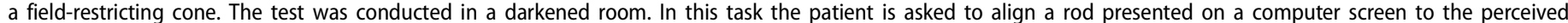

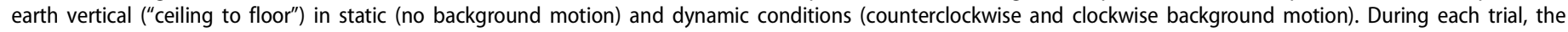

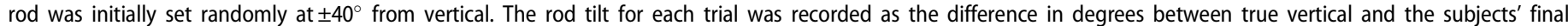

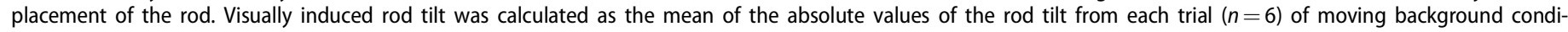

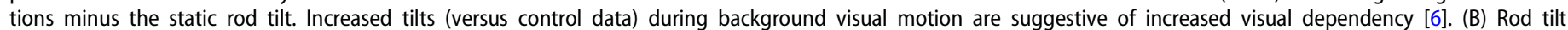

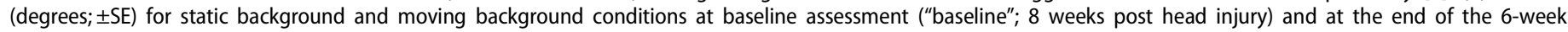

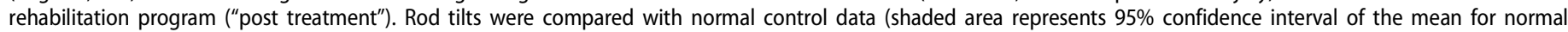
controls, from [6]).

and a formal neuro-ophthalmological assessment revealed only mild presbyopia that corrected with spectacles. Specifically, there was a full range of eye movements, no nystagmus, normal pursuit and saccades, and normal stereoscopic vision.

We quantitatively recorded his balance using the Berg Balance Scale, and functional outcomes via the Functional Independence and Functional Assessment Measures questionnaires. We undertook a bedside dynamic subjective visual vertical test (Figure 1(A)) using the rod and disk test (using his reading glasses), described in Cousins et al. [6] upon admission to the rehabilitation unit. He was not taking neurotropic medication.

Statistical analysis was carried out using SPSS v18 (Chicago, IL) and consisted of the Student $t$-tests for the rod and disk test (patient versus healthy controls), and Fisher's exact test (twotailed) for the questionnaire data (pre- versus post-rehabilitation comparisons).

\section{Rehabilitation}

The tailored rehabilitation program included input from neurophysiotherapists, occupational therapists, and neuropsychologists. The program focused on re-weighting and subsequently re-integrating ascending interoceptive information, via gradual removal of visual fixation strategies that the patient had developed to maintain balance. This was achieved by using activities without vision or with vision occluded (e.g., asking the patient to complete an activity whist holding a large gym ball in the visual field), challenging somato-sensation by using a variety of surfaces, and focusing on vestibular rehabilitation (e.g., by using head and whole body rotations in single and combined planes). Structuregraded exposure to real-life functional activities was guided by the goal-setting process (e.g., accompanied journeys on the London underground), and included both tasks of postural orientation and postural control [7].

\section{Results}

There was no change in static condition rod tilts between baseline and after rehabilitation (1.2 $2^{\circ}[\mathrm{SE} \pm 0.14]$ versus $1.0^{\circ}$ [SE \pm 0.2$]$; Student's t-test: $p=0.18$ ), and values were within $95 \%$ confidence intervals $(\mathrm{Cl})$ of control data [6]. However, the patient had significantly elevated visually-induced rod tilts at baseline (outside 95\% $\mathrm{Cl}$ of control data) that normalized after treatment $\left(6.9^{\circ}\right.$ [SE \pm 0.57 ] versus 3.13 $[\mathrm{SE} \pm 0.19]$; Student's t-test: $p<0.001$; Figure 1(B)). Following the rehabilitation program, improvement of visual dependency coincided with improvement in gait and balance observed clinically and semi-quantitatively with the Berg Balance 
Table 1. Clinical semi-quantitative outcome measures of balance function and functional independence on admission to rehabilitation unit, and after 6 weeks of focused rehabilitation of postural control.

\begin{tabular}{lccc}
\hline Clinical outcome measure & Pre-rehabilitation & Post-rehabilitation & $p$ Values \\
\hline Berg Balance Scale (0-56) & 18 & 54 & $<0.0001$ \\
FIM + FAM (30-210) & 173 & 204 & $<0.001$ \\
\hline
\end{tabular}

FIM: functional independence measure; FAM: functional assessment measure.

score $(p<0.0001)$, and both the Functional Independence and Functional Assessment Measures $(p<0.001$; Table 1).

\section{Discussion}

We highlight three observations of clinical interest: (1) visual dependency may contribute to gait and balance impairment following TBI; (2) its presence can be identified and its severity assessed using a simple bedside clinical test; (3) targeted physical therapy to reduce visual dependency can then be used to improve dizziness, gait and balance, and minimize long-term disability in some patients after TBI.

Humans are dependent on vision for accurate spatial orientation and navigation in the light. Visual dependency refers to an inappropriate reliance on visual input where it would be better to use proprioceptive or vestibular cues. Such situations arise where there may be a conflict between perceived external world motion and self-motion. The cardinal feature suggestive of abnormal visual dependency in our patient was abnormal trunk movement that was only observed in the context of optic flow (when the patient was walking or when there was external visual motion, such as passers-by) [8] that improved with eyes closed. We have previously shown a correlation between increased rod tilt and dizziness handicap inventory scores in patients with vestibular neuritis [6], further supporting a role for visual dependency in our patient's balance disturbance. Our patient's extreme but nonsustained multi-directional sway exhibited during stance suggested a functional (psychogenic) balance disorder [5,9]. Whilst many patients with $\mathrm{mTBI}$ (and also those with functional balance disorders) will volunteer troublesome symptoms of imbalance in the absence of objective neurological deficits [10], our patient with severe TBI was unaware of his postural impairment.

Benign paroxysmal positional vertigo is a common sequel of mild TBI [11]. Repeated visual motion stimulation induces adaptation in visual dependence following BPPV, so the presence of BPPV may explain why our patient developed abnormal levels of visual dependency [12]. It is theoretically plausible that in susceptible individuals, BPPV, even if treated early, could trigger a switch towards increased visual dependency. Whether prompt treatment with repositioning maneuvers reduces this tendency is not clear.

We observed clinical improvements in our patient's gait and balance with the rehabilitation program as evidenced by a higher Berg Balance score and his ability to return to commuting on public transport. There were also improvements in his overall function as documented in the FIM and FAM scores (Table 1). Gait impairment after TBI may result from impairment in a number of sensory modalities, including visual, vestibular, proprioceptive, and associated physical and emotional factors. Given that the rehabilitation program also involved neurocognitive and occupational therapy treatments, we cannot comment on which aspect contributed most to the change in visual dependency. Nevertheless, this case suggests that the identification of visual dependency, so that physical rehabilitation can be targeted, may avoid or reverse the development of mal-adaptive strategies which preclude normal everyday balance after not only mild but also moderatesevere TBI.

\section{Disclosure statement}

The authors have no conflicts of interest to report.

Ethics approval was obtained from Homerton University Hospital.

\section{References}

[1] Basford JR, Chou L-S, Kaufman KR, et al. An assessment of gait and balance deficits after traumatic brain injury. Arch Phys Med Rehabil. 2003;84:343-349.

[2] Kaufman KR, Brey RH, Chou L-S, et al. Comparison of subjective and objective measurements of balance disorders following traumatic brain injury. Med Eng Phys. 2006;28: 234-239.

[3] Williams G, Morris ME, Schache A, et al. Incidence of gait abnormalities after traumatic brain injury. Arch Phys Med Rehabil. 2009;90:587-593.

[4] Buster TW, Chernyavskiy P, Harms NR, et al. Computerized dynamic posturography detects balance deficits in individuals with a history of chronic severe traumatic brain injury. Brain Inj. 2016;30:1249-1255.

[5] Baik JS, Lang AE. Gait abnormalities in psychogenic movement disorders. Mov Disord. 2007;22:395-399.

[6] Cousins S, Cutfield NJ, Kaski D, et al. Visual dependency and dizziness after vestibular neuritis. PLoS One. 2014;9: e105426.

[7] Massion J. Postural control system. Curr Opin Neurobiol. 1994; 4:877-887.

[8] Guerraz M, Yardley L, Bertholon P, et al. Visual vertigo: symptom assessment, spatial orientation and postural control. Brain. 2001;124:1646-1656.

[9] Merello M, Ballesteros D, Rossi M, et al. Lack of maintenance of gait pattern as measured by instrumental methods suggests psychogenic gait. Funct Neurol. 2016;27:217-224.

[10] Honaker JA, Lester HF, Patterson JN, et al. Examining postconcussion symptoms of dizziness and imbalance on neurocognitive performance in collegiate football players. Otol Neurotol. 2014;35:1111-1117.

[11] Fife TD, Giza C. Posttraumatic vertigo and dizziness. Semin Neurol. 2013;33:238-243.

[12] Geurts AC, Ribbers GM, Knoop JA, et al. Identification of static and dynamic postural instability following traumatic brain injury. Arch Phys Med Rehabil. 1996;77:639-644. 